\title{
Using the Critical Path Method in Analyzing the Interdependencies of Critical Services - Feasibility Study
}

\author{
Andro Kull \\ Digital Safety Lab, Tallinn University, Tallinn, Estonia \\ Andro.Kulletlu.ee
}

\begin{abstract}
Everyday life is more and more dependent on critical services and no one can argue that interruptions in transportation, electricity, etc. cause big problems in everyday lives. The interdependencies inside of certain sector services are growing, for example financial services are interrupted in losing internet banking system. Also, the interdependencies between critical infrastructure (CI) services are growing - in case of the power failure, the financial services are not usable etc. The aim of this paper is to propose another approach to describe the interdependencies between critical services. There is a lot of research done in analyzing and modelling the interdependencies between critical services, for example a survey performed by Idaho National Lab under the sponsorship of U.S. Technical Support Working Group identified 30 tools for critical infrastructure interdependency modeling [1]. Our research is adding some specialties in modelling $\mathrm{CI}$ interdependencies.
\end{abstract}

Keywords: critical infrastructure, critical path method, interdependency analysis, recovery objectives.

\section{$1 \quad$ Introduction}

Under this research, a feasibility study is carried to find out if the theory called CPM critical path method [2] will be used on CI interdependency analysis. The essential technique for using CPM is to construct a model of the project that includes the following [3]:

- A list of all activities required to complete the project;

- The time (duration) that each activity will take to complete;

- The dependencies between the activities;

- Logical end points such as milestones or deliverable items.

During the study, two example cases are created to show the dependencies on critical service and analyze the most important characteristics using CPM.

As a result of this study, the main research question will be answered and the further research connects to development of CMP model for CI. The proposed model 
should be implemented as a solution, which allows simulations regarding recovery scenarios and give the basis for analyzing CI interdependencies.

\section{Overview}

In Estonia, the Emergency Act [4] has been adopted few years ago and during the practical implementation of the act, some shortcomings are occurred. The main points need clarification is related to how the components of CI interact and a more accurate definition of a vital service. European Programme for Critical Infrastructure Protection [5] says, that "The identification and analysis of interdependencies, both geographic and sectoral in nature, will be an important element of improving critical infrastructure protection in the EU. This ongoing process will feed into the assessment of vulnerabilities, threats and risks concerning critical infrastructures in the EU." The research results are meant for institutions in governmental level that are responsible to organize critical infrastructure protection by certain sector. Also, the real actions should be taken by critical service providers, who must be able to understand how their actions affect $\mathrm{CI}$ as a whole.

Current research is a part of research program and specific research topics under the program include, but are not limited to:

- Social studies - the mean for a vital service, what boundaries (time, scale, geographic indicator, etc.) becomes a vital service?

- Critical services impact studies - what will happen if the vital service availability is not guaranteed, what are the economic, political, social impacts in accordance with the length of the outage?

- Analysis of the dependencies between critical services - functional diagram or model, what kind of separate parts of a vital service consists of and how the interaction between these components provide a vital service operation?

- Analysis of risk scenarios - the most likely and / or a greater impact of the scenarios, the corresponding continuity capability assessments?

- Critical service operation objectives - their possible changes during a longer period (e.g., year, month, week, etc.).

Current paper is as introduction for analysis of the dependencies between critical services to find the suitable tools to carry out this analysis.

\section{Critical Infrastructure}

Critical infrastructure protection (CIP) is a concept that relates to the preparedness and response to serious incidents that involve the critical infrastructure of a region or nation [3]. In Estonia, there is not much statistics about critical infrastructure incidents and while the incidents happen, it is not hard to guess that in many cases the incidents are caused by failures of information systems. As a rule, the CI interdependencies are divided: physical, cyber, geographical, logical [6]. Current research focuses on cyber (dependency on information systems) and logical (time) dependencies. 
While getting the ability to model the dependencies between critical infrastructures, the practical problems and research questions will be asked from practicians who are CI services organizers or CI service providers. Further research includes the seminars and brainstorming to clarify the specific questions such as for example "What kind of problems related to the CI protection should the model allow to solve?"

In the area of critical infrastructure, the terms are used are mostly related to the business continuity (BC) like recovery time objective (RTO), recovery point objective (RPO), maximum tolerable period of disruption (MTPOD), business impact analysis (BIA) and key risk indicators (KRI) etc.

RPO EVENT

RTO

Fig. 1. Key terms on time scale

Business impact analysis helps us to find the events which may cause continuity disruptions of critical services. At the same time, key risk indicator lets us know if something is happened or there is high probability that something is going to happen. Maximum tolerable period of disruption gives the overall time frame, during which the recovery of critical service is meaningful.

In terms of dependencies analysis, we are concentrating to the right side of event in the case of service disruption as shown in Figure 1. Since we are focusing on time dependencies in our study, the RPO and other characteristics connected with resources are out of scope.

\section{Critical Path Method}

The critical path method (CPM) is an algorithm for scheduling a set of project activities [7]. CPM mostly deals with project modeling and management, in our case the project is treated as a set of recovery activities to ensure critical services continuity during predetermined time. As stated below, service recovery has the main project characteristics.

It was also considered during the study to use Critical Chain Project Approach (CCPA) instead of Critical Path Approach (CPA). Comparison between Critical Path and Critical Chain is stated by Critical Chain Ltd [8] and is provided in Table 1. In common, if we take the first criteria "The project finish is a date we think we can hit" (by CPA) and "The project finish is planned with a chosen level of likelihood" (by CCPA), the recovery project finish has to be clear and stated as RTO. Also, by recovering critical services, we have to consider that some of the project tasks may be taken by external service providers and to get those services, it needs to be agreed beforehand the exact criteria for these services. Therefore in comparing criteria "To keep the project on schedule, we must keep each task on schedule according to the calendar" (by CPA) and "To keep the project on schedule, we manage our buffers" (by CCPA) we have to choose the first one. 
Table 1. Comparison of CPA and CCPA

Critical Path Approach

\section{Critical Chain Project Approach}

The project finish is a date we think weThe project finish is planned with a chosen can hit (and then we work like hell tolevel of likelihood, and assured with buffers make it) throughout

The critical path determines the startThe critical path determines the end of the and end of the project - and the pathproject (after a project buffer is added to it), may change during the project but the start is often determined by a noncritical activity. The path does not change

Variation is implicit, and assumed to Variation is explicitly planned and managed "average out" over the length of thethroughout the project with buffers project

To keep the project on schedule, weTo keep the project on schedule, we manage must keep each task on schedule ac-our buffers, which allows us to absorb variacording to the calendar tion efficiently

Task start and finishes are carefullyBuffer status is carefully tracked. When any tracked. Schedule "slippage" is impor-task starts or finishes relative to the calendar is tant and must be monitored closely not important

People are evaluated in terms ofHalf of all tasks are expected to take longer whether their tasks are late relative tothan planned, and the buffers absorb such their committed calendar date for taskvariation

completion

Fixed-date "Stage gate" reviews areFloating "stage gate" reviews are triggered by scheduled to evaluate project progressphase completion, and buffer status is reto date viewed for project completion likelihood

The amount of slack that non-criticalNon-critical paths must have sufficient "feedpaths have is not as important and noting buffers" to protect the critical path tracked

Making progress on every project,Multi-tasking of resources is devastating, and during every reporting period, is im-is avoided at ALL costs, including delaying portant, so resources are multi-taskedthe start of projects to keep busy 
CPM is often used in conjunction with PERT - Project Evaluation and Review Technique [3]:

- PERT chart explicitly defines and makes visible dependencies (precedence relationships) between the work breakdown structure elements;

- PERT facilitates identification of the critical path and makes this visible;

- PERT facilitates identification of early start, late start, and slack for each activity;

- PERT provides for potentially reduced project duration due to better understanding of dependencies leading to improved overlapping of activities and tasks where feasible.

PERT terminology will be used further in analyzing the CPM and dependencies.

\section{$5 \quad$ Feasibility Study}

During the feasibility study, recovery of one certain service is taken as an example and it is described in context of ordinary project.

Every project has at least three main characteristics:

1. Purpose - what is the outcome of the project?

2. Time - how much it will take time?

3. Resources - how much resources are needed to achieve the results of the project within the given time?

In parallel, the recovery of any critical service needs to be answered:

1. Purpose - what level of service needs to be recovered?

2. Time - what is the recovery time objective (RTO)?

3 . Resources - what kind of resources are needed to recover predetermined level critical service within the given time?

In our case, the OpenProj (version 1.4) was used as project description software and the software was used in MacBook Air computer.

\subsection{Construction of Example Cases}

In constructing the example case, an ordinary IT service chain was used and it was divided into sub-services as shown in Figure 2. We have one scenario for disruption losing electricity and two possible scenarios for recovery - wait until electricity is back and start recovery in primary site or start recovering in alternative site, which in our case is so called cold site (basically it is only room with electricity and communications). The goal of this exercise is to analyze two possible recovery scenarios using critical path method in terms of electricity dependency and based on analyze, decide which scenario gives better results (it means shorter recovery time). 

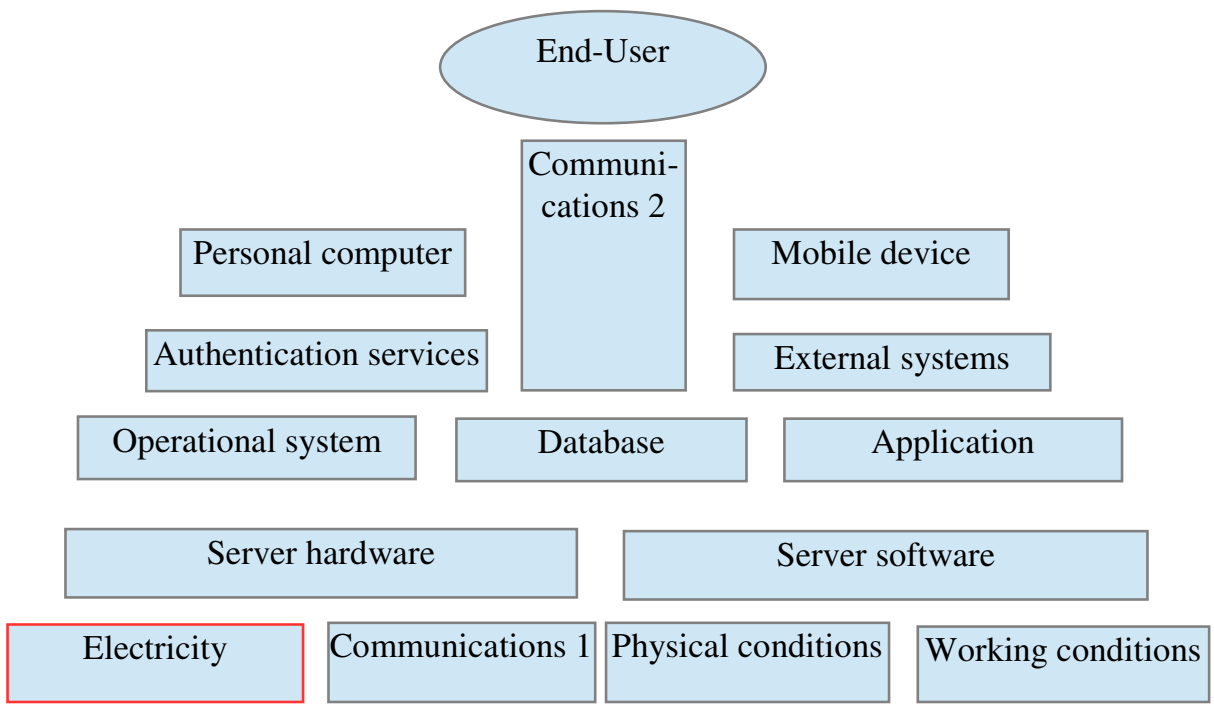

Fig. 2. IT service chain

Assuming, that our IT service is critical (based on BIA), we may expand research approach to dependency analysis between critical infrastructure services (CI).

\subsection{Finding Dependencies}

In Figure 2, the critical dependencies may be as following:

1. Dependency on electricity (critical service);

2. Dependency on communications (also critical service and seen in figure twice: basically, once needed for server-side and secondly needed for client-side);

3. Dependency on authentication service (for example in Estonia the IT services need to authenticate with ID card or mobile ID only);

4. Dependency on external systems (for example in Estonia the IT services need to communicate over X-road solution with national databases).

The recovery time objective (RTO) is the main indicator for creating plan for service (either critical or non-critical) recovery. Each task which needs to be recovered has its own RTO and combining the RTOs of each task allows us to measure the whole RTO of service. Now the need for critical path follows which allows us to find the critical tasks for service recovery. If there are tasks from other service providers (critical service providers), the criticality of such services and therefore also dependencies (including time dependencies) must be analyzed.

Taking all the tasks from critical path, assessment should follow which tasks are dependent on other (critical or not) service providers. The recovery time objective of each task in critical path and the resources needed to recover for the outsourced services have to be agreed by SLA-s (service level agreements). 


\subsection{Recovery Projects}

For recovery project, it is possible to create Gantt charts for scenarios to recover the IT service. For example, in case of electricity failure in primary site the Gantt chart may be drawn as in Figure 3.

For recovery project, it is possible to create Gantt charts for scenarios to recover the IT service. For example, in case of electricity failure in primary site, the Gantt chart for restoring IT service in secondary site may be drawn as in Figure 4.

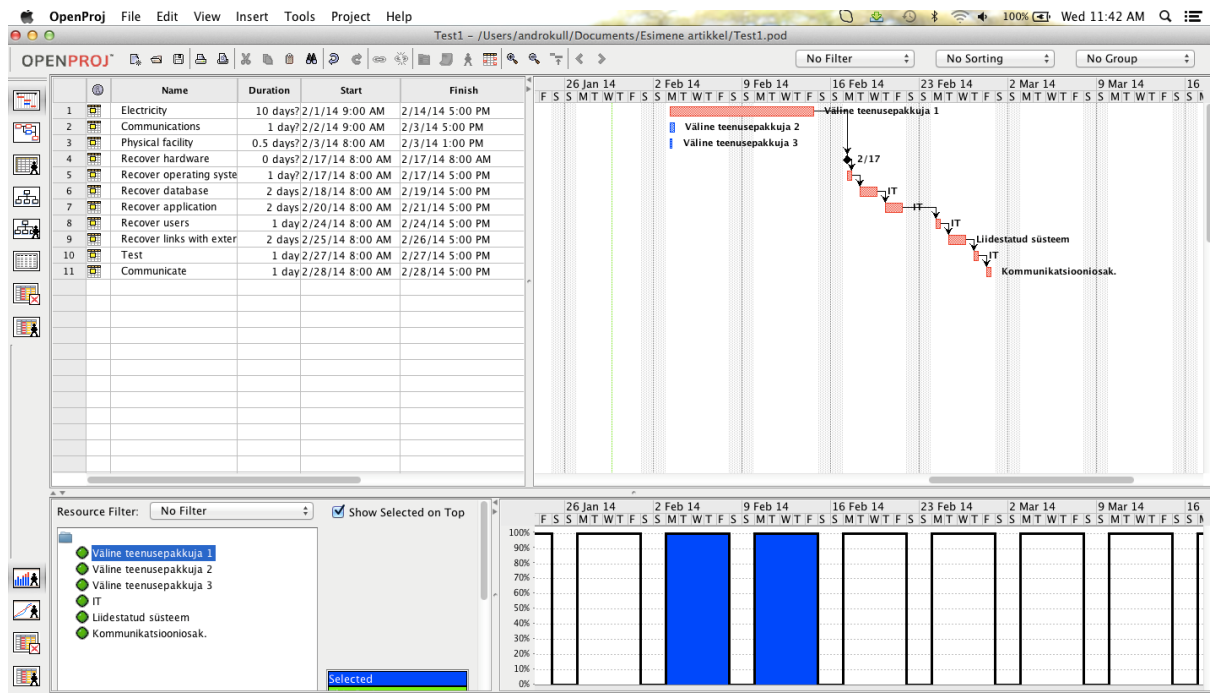

Fig. 3. Gantt chart for recovering in primary site (Test1.pod)

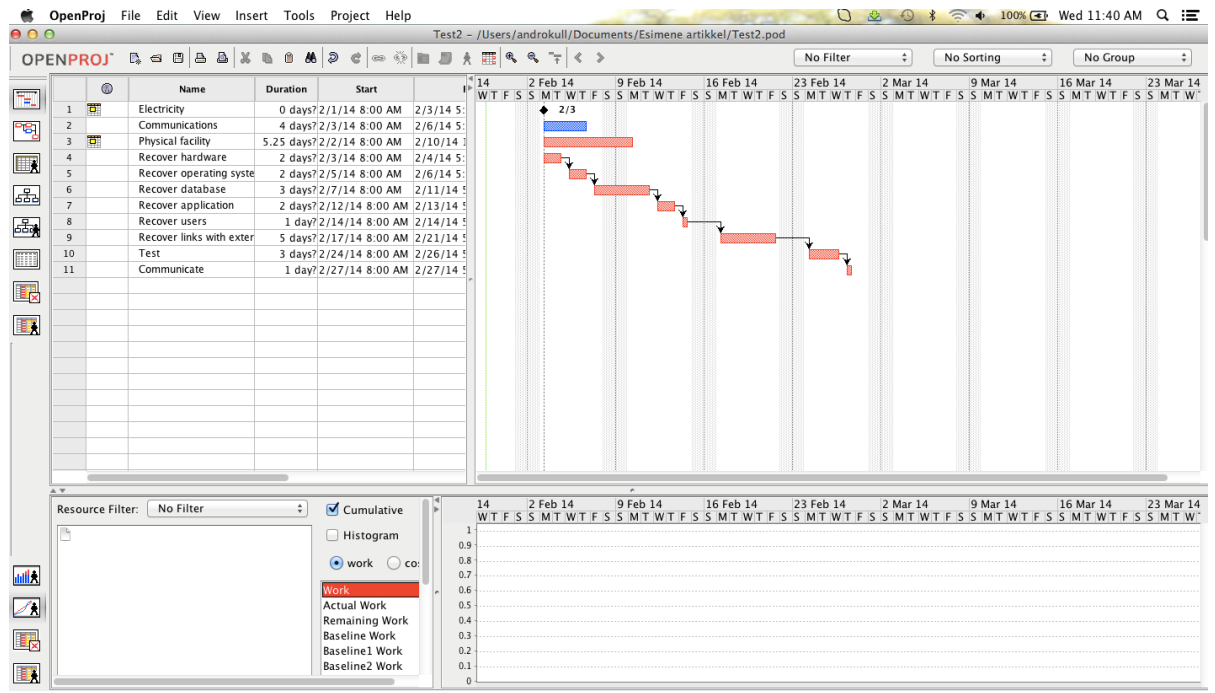

Fig. 4. Gantt chart for recovering in secondary site (Test2.pod) 
One shortcoming appears using normal project management tool (such as OpenProj used in our case) as it uses for duration days by default, but for example recovering IT system or services hours could be more suitable. To make situation visible and more actual, we may agree that column "duration" is not taken as days (in the figures) but as hours.

\subsection{Dependency Analysis}

To start analysis, the important characteristics used by the critical path method and clearly focusing on measuring time are as following: the duration, the early start (ES), the early finish (EF), the late start (LS) and the late finish (LF).

The most time consuming task in Test 1 is wait for electricity and in Test 2 prepare the secondary site.

In common, the duration of electricity failure determines the early start of recovering hardware. In recovering primary site, ES for recovering hardware is 10 hours (until electricity is assured) and in recovering secondary site, ES for recovering hardware (considering the dependency on electricity) is in principle 0 hours. Still, the option for starting recovering in secondary site is not obvious and may be dependent on other facts, which has to find out in answering the questions like "Do we keep necessary hardware in secondary site?" or "How long it takes to transport necessary hardware to secondary site?". Answering those questions, the real ES for hardware recovery is possible to identify.

Also, we assume in Test 2 that necessary component in IT service chain - communications - recovery may start immediately because there is necessary electricity for devices (i.e., the ES for communications is 0 hours), in Test1 all necessary steps to recover communications without electricity (i.e., everything is up and running immediately when electricity is back) may not actually be possible and we cannot assume that duration of recovering communications is 0 hours.

One may argue that in our example case, for electricity recovery may use the following categories as stated by PERT [3]:

- optimistic time $(\mathrm{O})$ : the minimum possible time required to accomplish a task, assuming everything proceeds better than is normally expected

- Pessimistic time $(\mathrm{P})$ : the maximum possible time required to accomplish a task, assuming everything goes wrong (but excluding major catastrophes).

- Most likely time (M): the best estimate of the time required to accomplish a task, assuming everything proceeds as normal.

- Expected time (TE): the best estimate of the time required to accomplish a task, accounting for the fact that things don't always proceed as normal.

And to find the most expected time when electricity is back, use the calculation

$$
\mathrm{TE}=(\mathrm{O}+4 \mathrm{M}+\mathrm{P}) \div 6
$$

To answer, we have to go back to the purpose of dependency research and in our case, the business continuity plan or recovery policy may assume that in recovering 
electricity-dependent IT services, we have to wait until indicated time (keep position to start recovering in primary site) and after indicated time start recovering in secondary site. That time is possible to set up by CPM analysis and calculating the best way to recover (i.e. the shortest recovery time). Basically it means that in recovering critical services, there should not be used terms like "expected".

\subsection{Implications for Practice}

In analyzing the critical path, we have to consider the duration of each critical service (in our terms, it is RTO for critical service) and we can calculate ES, EF, LS and LF for each critical sub-service in critical path.

By the CPM, the recovery time for sub-services provided by external service providers may be calculated and it should be controlled against the service provider continuity objectives (RTO). Usually these objectives are agreed as SLA (Service Level Agreement). Besides of RTO for critical service which may be agreed by the contract between critical service provider, it has to be also agreed and tested, if the service level meets our ES, EF, LS and LF targets. To find out these targets, CPM is visible.

Based on feasibility study it became clear that CPM may be simple and powerful tool to analyze different solutions for critical services recovery and thereby analyze the interdependencies between critical infrastructure services. Based on analysis, the important questions for setting up the recovery strategies or business continuity plans are to be answered.

\section{Conclusion}

In order to create recovery plan (or project in our case) for IT service, we have to first decide recovery time objective based IT service criticality for end-user, specify the sub-services needed to get IT service up and running and also there is need to describe somehow the interdependencies on critical services which are not under our control. The overall purpose of feasibility study was to find the critical path for IT service recovery and show the dependencies on critical sub-services. Based on feasibility study, it was found out that the critical path method is usable to analyze critical infrastructure dependencies.

The further research connects to development of CPM model for CI and the proposed model will be implemented as a solution, which allows simulations regarding risk scenarios and give the basis for CI recovery scenarios. Based on our example, this solution will allow to make analyzed decisions about for example if there is a need for alternative warm site to recover IT service and thereby shorten recovery time objective, maybe the acceptable solution is to review the service levels with critical service providers etc. 


\section{References}

1. Pederson, P.: Critical Infrastructure Interdependency Modeling: A Survey of U.S. and International Research, http: / /www.inl.gov/technicalpublications / Documents/3489532.pdf

2. Kelley, J., Walker, M.: Critical-Path Planning and Scheduling. In: Proceedings of the Eastern Joint Computer Conference (1959)

3. Wikipedia, http://www.wikipedia.org

4. Teataja, R.: Emergency Act (Hädaolukorra seadus), https://www.rigiteataja.ee/akt/130102012003

5. European Commission: European Programme for Critical Infrastructure Protection (EPCIP), http: / / europa.eu/legislation_summaries / justice_freedom_security/fight_against_terrorism/133260_en.htm

6. Rinaldi, S.: Identifying, Understanding, and Analyzing Critical Infrastructure Interdependencies. IEEE Control Systems Magazine (December 2001)

7. Kelley, J.: Critical Path Planning and Scheduling: Mathematical Basis. Operations Research 9(3) (May-June 1961)

8. Critical Chain Ltd., http: / /www. criticalchain.co.uk/ 